\title{
Investigation of Noise Spectrum Characteristics for an Evaluation of Railway Noise Barriers
}

\author{
Hyo-In Koh ${ }^{\dagger}$, Seungho Jang* and Ji-Young Hong*
}

\begin{abstract}
Most of the test methods used for determining the acoustic performance of the noise barriers in Korean standards address the sound-proof panels but refer to noise barriers as a whole system or their in-situ performances less. Many new types of trains with different operating speeds have been developed and produced, and civil appeals against railway noise are becoming quite extensive. Considering these latest changes of circumstances, it is necessary to investigate the current standards and specifications pertaining to railway noise barriers. In this paper, criteria for the measurements and evaluations of noise barriers and sound-proof panels are examined and experimental studies are introduced. In order to suggest more efficient methods for reasonable evaluations of the noise barriers, frequency spectrums of the noise source, the power of the actual railway vehicles, are investigated and introduced.
\end{abstract}

Keywords : Noise barrier, Railway noise, Frequency spectrum, Measurement

\section{Introduction}

\subsection{Standards for noise barriers}

In Korea, most noise barriers are tested and evaluated primarily according to KS(Korean Standards), which deal with the quality conditions of noise-proofing panels, such as acoustical as well as non-acoustical properties. Regarding performance tests for the in-situ evaluations, ISO 10847 is adopted as an ISO KS 10847 regulation. In addition, the Ministry of the Environment and the Seoul Metropolitan Government established regulations pertaining to noise barriers, with which the installation, performance criteria, and maintenance operations are carried out [1,2].

In 2000, standard regulations for the railway noise barriers were proposed through research [3] by the Korea Railroad Research Institute. In this research, normalized frequency spectrums were introduced. Based on the spectrum characteristics, it was suggested that these frequency bands should all be included in the evaluation process of

\footnotetext{
Corresponding author: Korea Railroad Research Institute, Korea E-mail : hikoh@krri.re.kr

Korea Railroad Research Institute, Korea

(c) The Korean Society for Railway 2013 http://dx.doi.org/10.7782/IJR.2013.6.3.125
}

absorption measurements of noise-proofing panels (Table 1). In addition, it was suggested that this normalized frequency spectrum of railway noise should be used as a reference source signal for in-situ measurements during performance evaluations of railway noise barriers.

Considering new railway vehicles types such as highspeed trains and light-weight trains and new track types forms, all built and introduced after the normalized sound spectrum was introduced in 2000 , it is urgently needed to redefine the technical meaning of the descriptor 'normalized' in 'normalized railway noise spectrum' and then to propose an updated normalized railway noise spectrum for Korean railways. In 2006 the Korea Rail Network Authority introduced specifications for high-speed railway noise barriers; this document deals with guidelines for the manufacturing, installation and performance of high-speed rail-

Table 1 Technical criteria pertaining to noise barriers performances

\begin{tabular}{ccc}
\hline Test item & Performance criterion & Standards \\
\hline $\begin{array}{c}\text { Airborne sound } \\
\text { insulation }\end{array}$ & $\begin{array}{c}\geq 25 \mathrm{~dB}(500 \mathrm{~Hz}) \\
\geq 30 \mathrm{~dB}(1 \mathrm{kHz})\end{array}$ & KS F 2808 \\
\hline Sound absorption & $\begin{array}{c}\mathrm{NRC} \geq 0.7(250 \mathrm{~Hz}, 500 \mathrm{~Hz}, \\
1000 \mathrm{~Hz}, 2000 \mathrm{~Hz})\end{array}$ & KS F 2805 \\
\hline
\end{tabular}


Table 2 Technical criteria pertaining to noise barriers performances for high-speed trains (Specification for highspeed railway noise barrier, Korea Rail Network Authority 2006)

\begin{tabular}{ccccccc}
\hline frequency $(\mathrm{Hz})$ & 125 & 250 & 500 & 1000 & 2000 & 4000 \\
\hline absorption & 0.5 & 0.8 & 0.9 & 0.95 & 0.9 & 0.7 \\
\hline
\end{tabular}

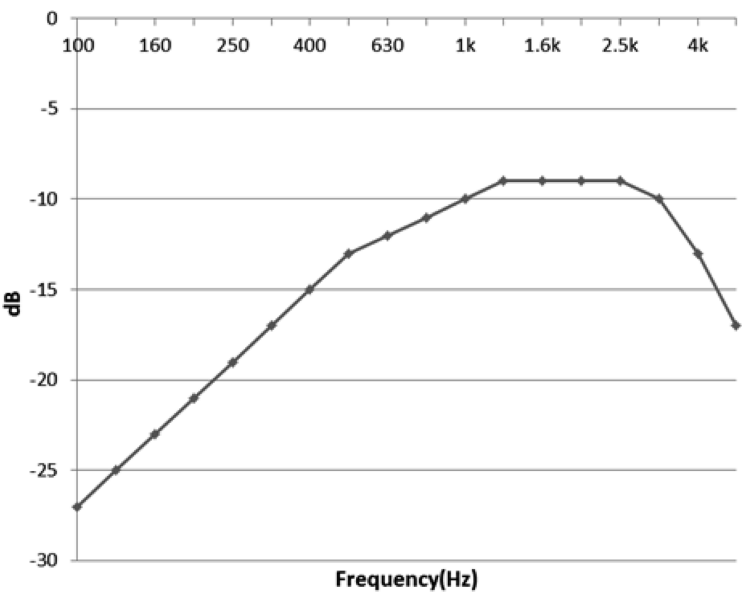

Fig. 1 Normalized Railway noise spectrum [4]

way noise barriers, also stipulation the requirement of technical criteria pertaining to acoustical performance from the CEN (European Committee for Standardization) (Table 2).

\subsection{Normalized railway noise spectrum}

In BS EN 16272-3-1 : 2012 [4], a normalized railway noise spectrum is specified for evaluations and assessments of the acoustical performances of devices designed to reduce airborne railway noise near railways, using singlenumber ratings for sound absorption and airborne sound insulation. The spectrum is expressed in terms of relative Aweighted sound pressure levels in decibel, for one-third octave bands in the frequency range of $100 \mathrm{~Hz}$ to $5 \mathrm{kHz}$.

In Korea, the normalized noise spectrum was investigated in 2000[3] for two types of rolling stock, Mugunghwa and Saemaul vehicles. In 2012[5], the noise spectrum of high- speed trains behind a noise barrier was determined for an assessment of the acoustical performance of a noise- reducing device attached onto the edge of a noise barrier. The frequency spectra were obtained by means of the average values of the measured sound pressure levels from two to four railway sites.

The average values of each frequency band were then normalized according to the value at $1 \mathrm{kHz}[3,5]$. The frequency-weighted spectrum was used to compensate for the results of the assessment of the acoustic performances of

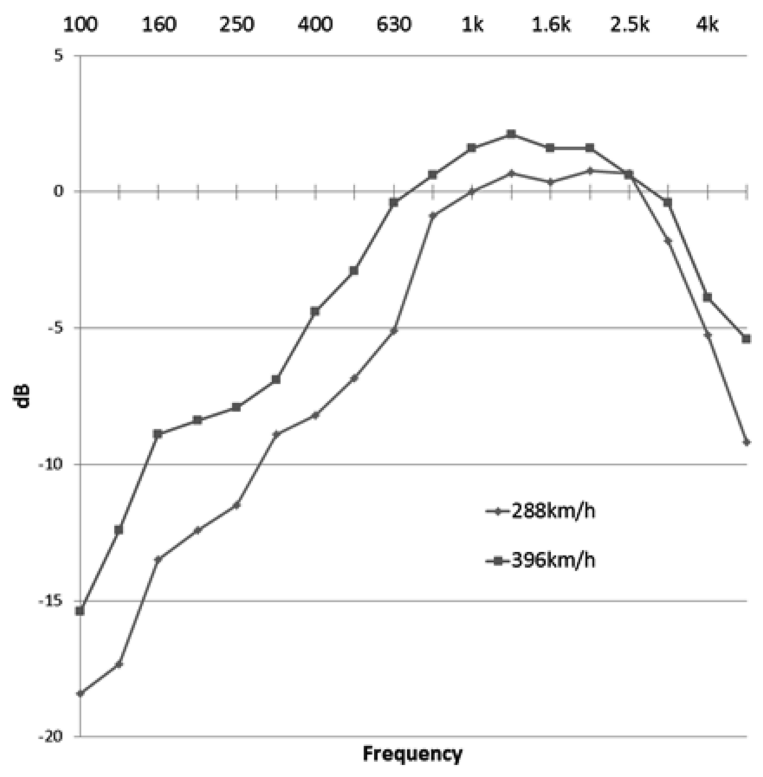

Fig. 2 Normalized spectral values of Korean high-speed trains $(288 \mathrm{~km} / \mathrm{h}, 396 \mathrm{~km} / \mathrm{h})$ measured on a slab track

noise barriers and noise-reducing devices.

In Fig. 1, the normalized railway noise spectrum specified in the BS EN 16272-3-1: 2012 [4] is shown. Additionally in Fig. 2, the measured sound pressure levels (dBA) of Korean high-speed trains on slab tracks are depicted. Here the relationship to the normalized railway noise spectrum proposed in the BS EN 16272-3-1 : 2012 [4] can be observed .

The difficulties in determining the technical criteria pertaining to the acoustical performance of railway noise barriers stem from the various noise sources of different types of vehicles and different measurement results from different sites. Unlike road transportations, there are for example railway tracks only for specific types of vehicles, specifically tracks for high-speed trains and or tracks for light-weight trains. As stated at the beginning of this paper, a normalized noise spectrum represents fundamental information for the manufacturing of noise barriers and for an assessment of the performance of noise barriers. In this paper, methodologies for the determination of the representative noise spectrum for railways are investigated and the results are shown and discussed.

\section{Determination of the Railway Noise Spectrum}

\subsection{Calculation of the noise levels using a prediction model}

In order to determine the representative noise levels of 


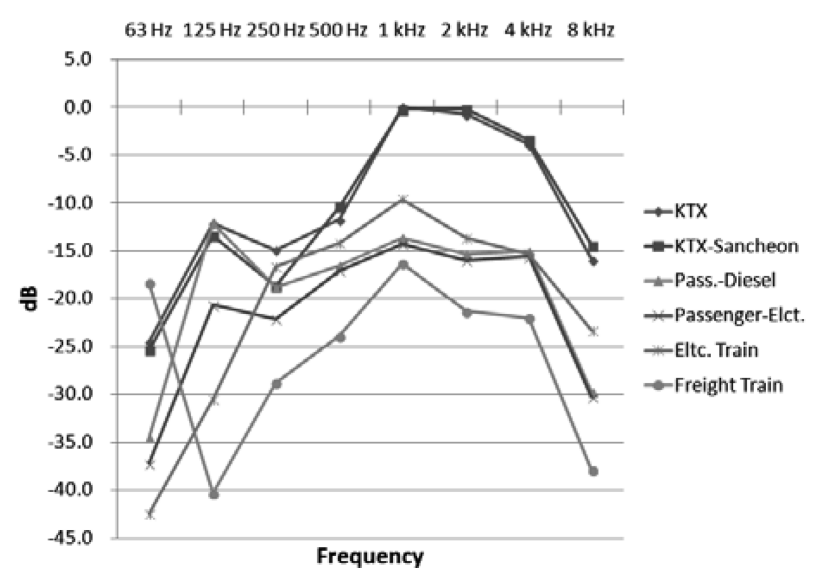

Fig. 3 Calculated and normalized sound level(TEL) of different vehicles at a distance of $7.5 \mathrm{~m}$ from the nearest rail

each type of railway vehicle, a railway noise prediction model is used which has been developing since 2009 by the Korea Railroad Research Institute [6,7]. This model was created from a combination of theoretical calculating model of wheel/rail rolling noise and a statistical analysis of various in-situ measurements.

In this model, the roughness characteristics of the rail and the wheels, the track conditions, a dynamic analysis of the contact between the wheels and the rails, the decay rate of the track, the train speed, and vehicle types are needed to calculate the sound power of the vehicle. The directivities of the sound propagation and attenuation due to distance and ground conditions are considered for the calculation of the sound propagation in the relevant paths. The sound pressure level is determined as follows:

$$
\begin{aligned}
& L_{e q, f, m}=L_{w, f, m}+D_{l}+10 \log \left(\frac{l_{m}}{l_{0}}\right)-A_{d i v, m}-A_{a, m} \\
& -A_{g r, f, m}-A_{b a r, f, m}
\end{aligned}
$$

Here, $L_{w, f, m}$ refers to the radiated sound power level due to a train passing-by in the $\mathrm{m}$ sector for frequency band $f$, $D_{l}$ is the directivity index, $l$ refers to the length of the considering track, $A_{d i v, m}$ is the attenuation due to geometrical divergence, $A_{a, m}$ is the attenuation due to atmospheric absorption, $A_{g r, f, m}$ is the attenuation due to the ground effect, and $A_{b a r, f, m}$ is the attenuation due to a barrier according to ISO 9613-2(1996)[8]. Fig. 3 shows the calculated noise level in $\mathrm{dB}(\mathrm{A})$ at a distance of $7.5 \mathrm{~m}$ from the rail. The sound levels are normalized to the values in the frequency band of $1 \mathrm{kHz}$. The train speed for high-speed trains is $305 \mathrm{~km} / \mathrm{h}$ and the train speed for passenger cars is $140 \mathrm{~km} / \mathrm{h}$, while it is $110 \mathrm{~km} / \mathrm{h}$ for metro trains and $80 \mathrm{~km} /$ $\mathrm{h}$ for freight trains. For all cases, ballasted track systems are

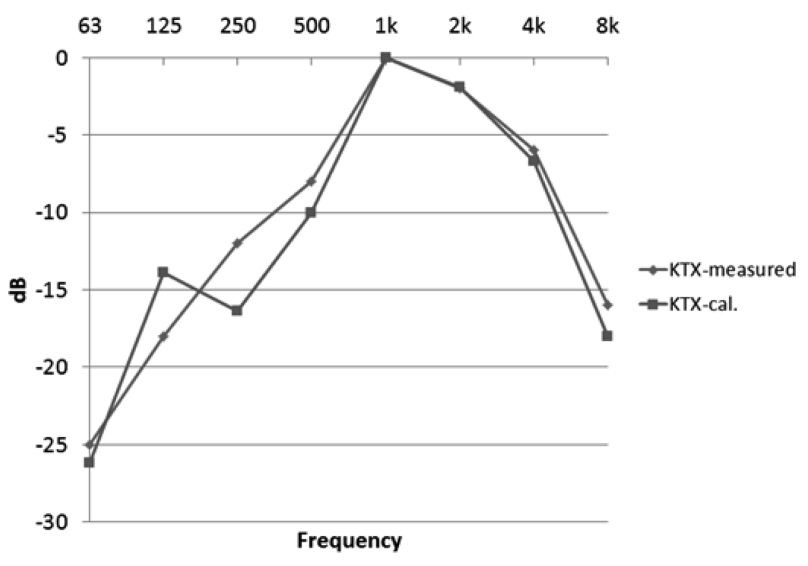

Fig. 4 Calculated and normalized sound levels of the KTX train $(293 \mathrm{~km} / \mathrm{h})$ at a distance of $7.5 \mathrm{~m}$ from the nearest rail

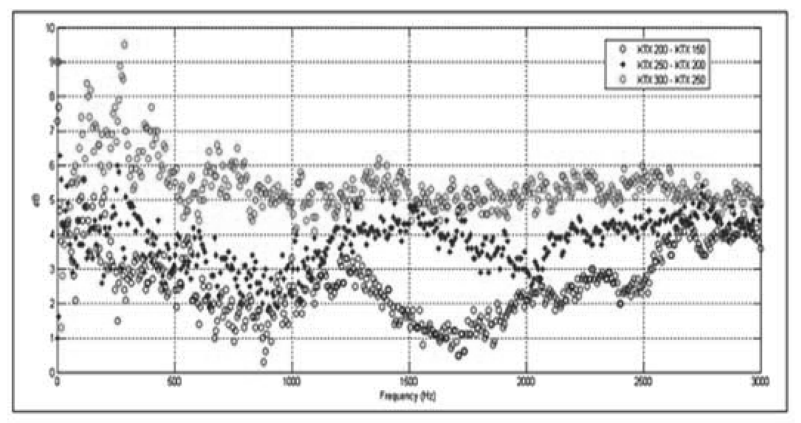

Fig. 5 Differences $(\mathrm{dB})$ in the measured noise levels of highspeed trains (ballast track $150 \mathrm{~km} / \mathrm{h} \sim 300 \mathrm{~km} / \mathrm{h}$ ) [7]

chosen. Fig. 4 shows the calculated values compared with the values from field measurement of the of the KTX train. In the lower frequency range, there exist some differences, while above $1000 \mathrm{~Hz}$, the measurement and calculation results show more similarity. This output relies on a variety of noise situations of KTX trains in the fields.

Fig. 5 shows the differences between the measured sound pressure levels of high speed trains. In the range of train speeds of $250 \mathrm{~km} / \mathrm{h} \sim 300 \mathrm{~km} / \mathrm{h}$, the differences in $\mathrm{dB}$ are quite large, with the maximum value exceeding 9 $\mathrm{dB}$. Considering the major deviations of the noise levels between vehicle types and between the measurements of the same types of vehicles, for an evaluation of the acoustic performance of noise-proofing panels or noise barriers, it can be more useful to use predicted noise levels than to use normalized weighting values determined through the average value of several different measurement locations.

\subsection{Determination of the noise source characteristics}




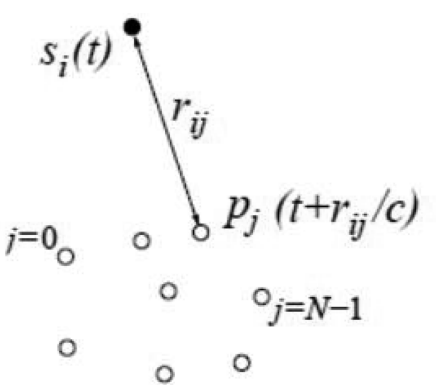

Fig. 6 Sound source Si with array of N sensors

For the laboratory test of the noise-proofing panels, sound absorption and airborne sound insulation assessments were done. However, in order to plan and build a noise barrier in the fields according to the requirements, the sound power distribution of the source and the radiation characteristics of the individual sources are also important, as sound experiences not only absorption or transmission but also diffraction and interference as it propagates through and around a noise barrier.

These characteristics are dependent on the frequency and the incidence angle relative to the noise barrier. The contribution of the noise sources become more complicated with high-speed trains due to the increasing aerodynamic sources at train speeds of about $250 \mathrm{~km} / \mathrm{h}$ and more. These include the pantograph, bogies, the louvers, inter-coach spacings and the front-rear nose parts. The locations of the noise sources and the acoustical properties can be investigated using microphone arrays or intensity arrays. Combining signal process technology methods such as beamforming in which a delay- and-sum algorithm, moving sources can be localized, separated and the source strengths can be measured. A point monopole sound source radiates into free air and a wave, emitted at time $t$, travels a distance $r$ with a speed of $c$ to the observation point. It arrives at time instant $t+r / c$. With an array of microphones, it is possible to focus on a specific point source by summing up all microphone signals $p$, accounting for propagation time delays between source and receivers.

$$
s_{i}(t)=\frac{\sum_{j=0}^{N-1} w_{j} r_{i j}(t) p_{j}\left[t+r_{i j}(t) / c\right]}{\sum_{j=0}^{N-1} w_{j}}
$$

$w_{j}$ are amplitude weighting factors (shading or tapering) (Fig. 6).

It is a typical problem with this type of technology however, that it is not obvious how to transform sound maps into absolute sound power levels of the different sources. This is due to the limited resolution, dynamic range and the beam width, as all practical array measurement results

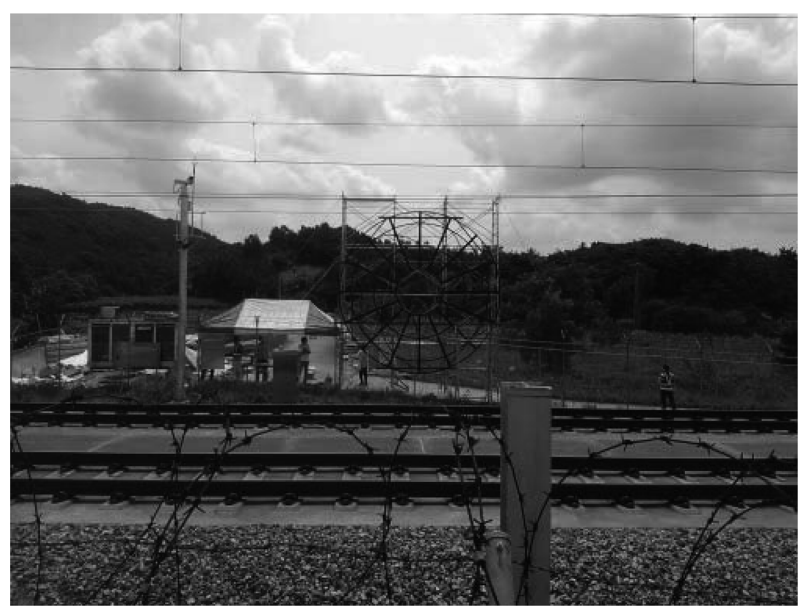

Fig. 7 Microphone array system

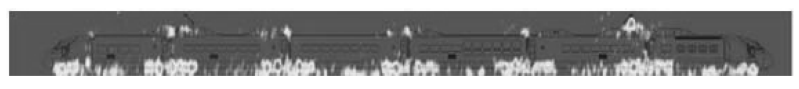

Fig. 7 Noise source map of a high-speed train $(350 \mathrm{~km} / \mathrm{h}, 630 \mathrm{~Hz})$

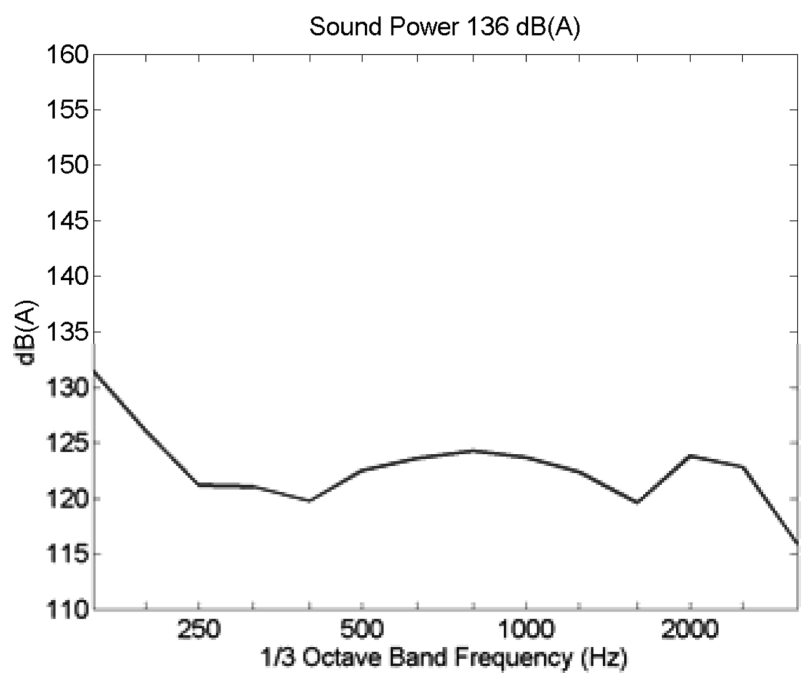

Fig. 8 Sound power of a whole high-speed train $(350 \mathrm{~km} / \mathrm{h})$

necessarily undergo deviations from results obtained by a perfect array, i.e. one with very small microphone spacings, and very large lengths/diameters [7]. The Korea Railroad Research Institute developed a microphone array measurement system with an optimal sensor configuration and analysis program.

The sound power calculation process is compensated for and updated through the measured transfer function between the moving source and the array and by means of a timesignal-simulation algorithm. In Fig. 7 the measurement system is shown and the sound sources are depicted for a high- 


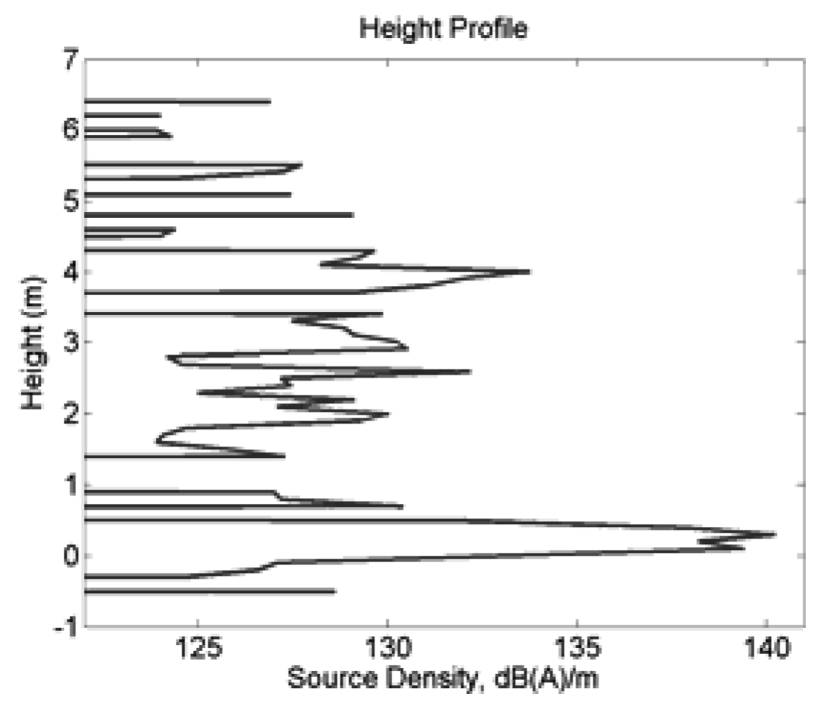

Fig. 9 Sound power of a high-speed train ; height profile $(350 \mathrm{~km} / \mathrm{h})$

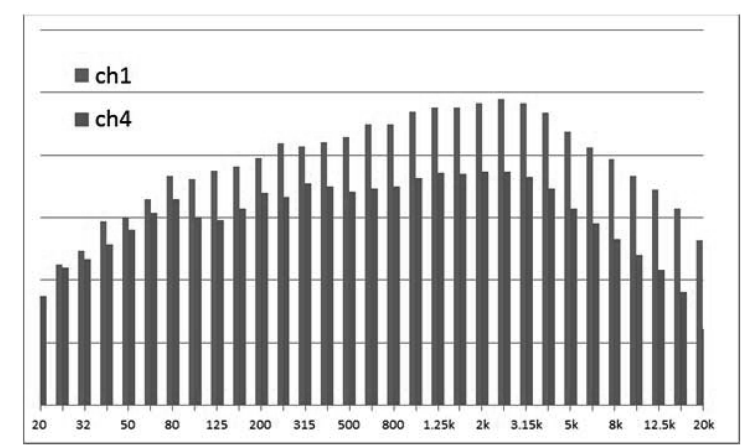

(a)

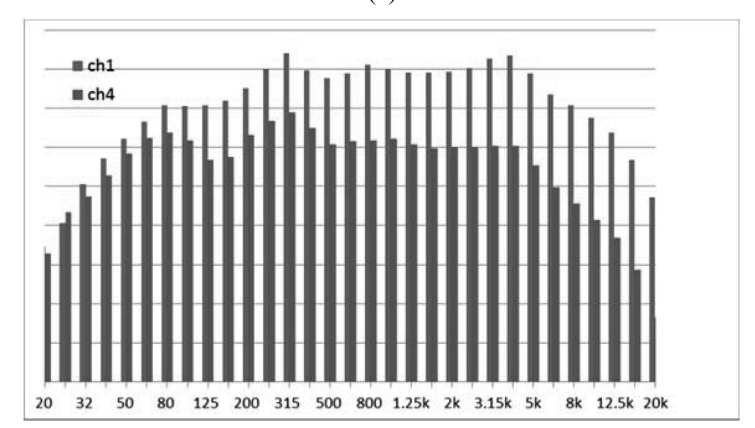

(b)

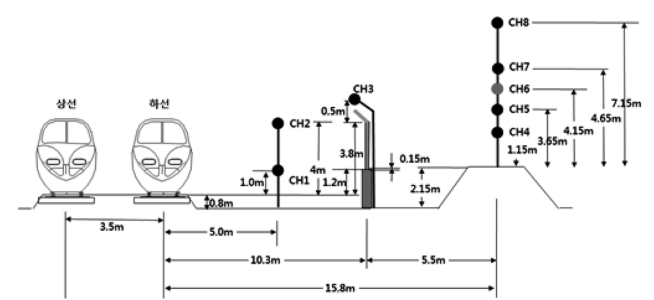

Fig. 10 Noise level measured at a noise barrier section along a high- speed line $(300 \mathrm{~km} / \mathrm{h})$;

front power car(a), passenger coach(b) speed train with a train speed of $350 \mathrm{~km} / \mathrm{h}$ (Fig. 8).

Fig. 9 and Fig. 10 show the radiated sound power of a high-speed train at a speed of $350 \mathrm{~km} / \mathrm{h}$.

\subsection{Noise level behind a noise barrier}

Fig. 11 shows an example of the measured noise level of a high-speed train, where noise barriers are installed. The radiated sounds of the passenger cars and the power cars show different frequency characteristics. When passenger cars pass by, the frequency area of $125 \mathrm{~Hz} \sim 500 \mathrm{~Hz}$ is as dominant as that in the range of $1000 \mathrm{~Hz} \sim 3000 \mathrm{~Hz}$. The sound energy of this low frequency part remains, whereas the sound of the higher frequencies is shielded by the noise barrier. When the power cars pass by, $1250 \mathrm{~Hz}$ $3150 \mathrm{~Hz}$ is the mainly radiated sound frequency area and this part contributes most to the sound behind the noise barrier. The sound power values along the vehicle height in Fig. 10 can be the useful information when planning and building an effective noise barrier near actual tracks.

Fig. 10 Noise level measured at a noise barrier section along a high- speed line $(300 \mathrm{~km} / \mathrm{h})$; front power car(a), passenger $\operatorname{coach}(b)$,

\section{Summary}

In this paper, the primary aim was to propose the noise characteristics of the railways which are intended to be used commonly for the determination of the acoustical performance criteria of noise barriers. Another aim was to evaluate the insertion losses of noise barriers and of related noise reduction devices. This is specified as a normalized railway noise spectrum in the BS EN standard which is to be used for the weighting of evaluation results and for evaluations with single-number rating. Due to the realistic conditions of each railway site and the different noise patterns at each, studies considering many aspects are needed for the efficient planning of various barriers and for the determination of the performance criteria for field tests.

\section{Acknowledgement}

This study was carried out within the framework of the research project 'A Study of the improvement of the Certification system and the Readjustment of Test Standards Related to Rolling Stock/Track/Environment Fields for Railway Product Parts.' funded by the ministry of Land, Infrastructure and Transport of Korea. The authors gratefully acknowledge the support for this research.

\section{References}


Hyo-In Koh, Seungho Jang and Ji-Young Hong / IJR, 6(3), 125-130, 2013

1. Ministry of Environment Korea (2011-135), Standard for the installation and performance of the noise barrier.

2. Seoul Metropolitan Government (2005), Guideline for the noise barrier and performance criterion.

3. Korea Railroad Research Institute (2000), A research on railway noise and vibration reduction.

4. BS EN 16272 : Railway Track Noise Barriers (2012).

5. Kim, C. H., Chang, T. C., Kim, D. S., Cho, J. H. (2012). "Performance Comparison of Noise Reducing Devices for the Different Noise Source Spectrum," Proceedings of the
Korean Society for Noise and Vibration Engineering Conference, Oct. pp.196-197.

6. Korea Railroad Research Institute (2012-2013), Prediction and Reduction of the Railway noise and vibration.

7. Korea railroad research institute (2010-2014), Development of Technologies for $400 \mathrm{~km} / \mathrm{h}$ noise source model and contribution.

8. ISO 9613-2 (1996), Acoustics-Attenuation of sound during propagation outdoors, part 2 : General method of calculation. 